\title{
Luminosity measurements and diffractive physics in ATLAS
}

\section{Christophe Royon*}

DAPNIA-SPP, CEA Saclay, F91191 Gif-sur-Yvette, France

E-mail: royon@hep.saclay.cea.fr

\begin{abstract}
We first describe the measurement of the elastic scattering in the Coulomb region which will be used by ATLAS to measure the absolute luminosity, as well as the LUCID detectors which allow to make a measurement of the relative luminosity. We also describe the project to build new roman pots at 220 meters and their Silicon strip and precise timing detectors allowing a resolution of $15 \mu \mathrm{m}$ and 5 ps respectively.
\end{abstract}

International Workshop on Diffraction in High-Energy Physics -DIFFRACTION 2006 -

September 5-10 2006

Adamantas, Milos island, Greece

*Speaker. 


\section{Luminosity measurement in the ATLAS collaboration}

It is important to be able to perform a measurement of the luminosity inside the ATLAS collaboration with great precision since it represents one of the main uncertainties for many measurements such as the measurement of the Higgs boson production cross section or of the value of $\tan \beta$.

\subsection{Different luminosity measurements}

There are two kinds of luminosity measurements: an absolute value which serves as a point of reference, and a relative one which allows to follow the values of the luminosity accumulated by the ATLAS experiment as a function of time. This last measurement is performed using a special detector called LUCID which we will describe in the following. We will concentrate now on an absolute measurement of the luminosity.

The goal of the absolute measurement is to reach a precision of about 2 to $3 \%$. It is possible to use different methods. The first one relies on the measurement of the LHC beam parameters. This corresponds to extrapolate some measurements performed by the LHC machine group outside the experimental area and the expected accuracy is of the order of 5 to $10 \%$, improving with time. The second method is to use a known QED or QCD cross section such as the production of a muon pair by double photon exchange (unfortunately, a small observable cross section), and $W$ production (which suffers from theoretical uncertainties of the order of 5\% which will decrease when NNLO calculations will be available). The third method is to use the optical theorem as in TOTEM, but this requires a good coverage of the full rapidity region which is not ideal for ATLAS. The fourth method which we will discuss in more detail in the following is based on Coulomb scattering.

\subsection{Measurement of elastic scattering in the Coulomb region}

When $t$ (the transferred energy at the proton vertex) is close to 0 , the $t$ dependence of the elastic scattering cross section reads:

$$
\frac{d N}{d t}(t \rightarrow 0)=L \pi\left(\frac{-2 \alpha}{|t|}+\frac{\sigma_{t o t}}{4 \pi}(i+\rho) e^{-b|t| / 2}\right)^{2}
$$

From a fit to the data in the Coulomb region close to $t \sim 0$, it is possible to determine directly the total cross section $\sigma_{t o t}$, the $\rho$ and $b$ parameter as well as the absolute luminosity $L$ [1]. This measurement requires to go down to $t \sim 6.510^{-4} \mathrm{GeV}^{2}$, or $\theta \sim 3.5 \mu \mathrm{rad}$ (when the strong amplitude equals the electromagnetic one). The UA4 collaboration already performed such a measurement [2] at the SPS and reached a precision of the oder of 3\%. However, let us note that the UA4 experiment needed to go down to $120 \mu \mathrm{rad}$ whereas the ATLAS collaboration needs to go down to 3.5 $\mu \mathrm{rad}$, which makes this measurement very challenging. This measurement requires a special high $\beta^{*}$ optics, detectors to be installed at about $1.5 \mathrm{~mm}$ from the LHC beam axis, a spatial resolution of these detectors well below $100 \mu \mathrm{m}$ and no significant edge on the detectors (less than $100 \mu \mathrm{m}$ ). The solution to achieve this measurement is to install roman pot detectors which can go close to the beam when the beam is stable. 

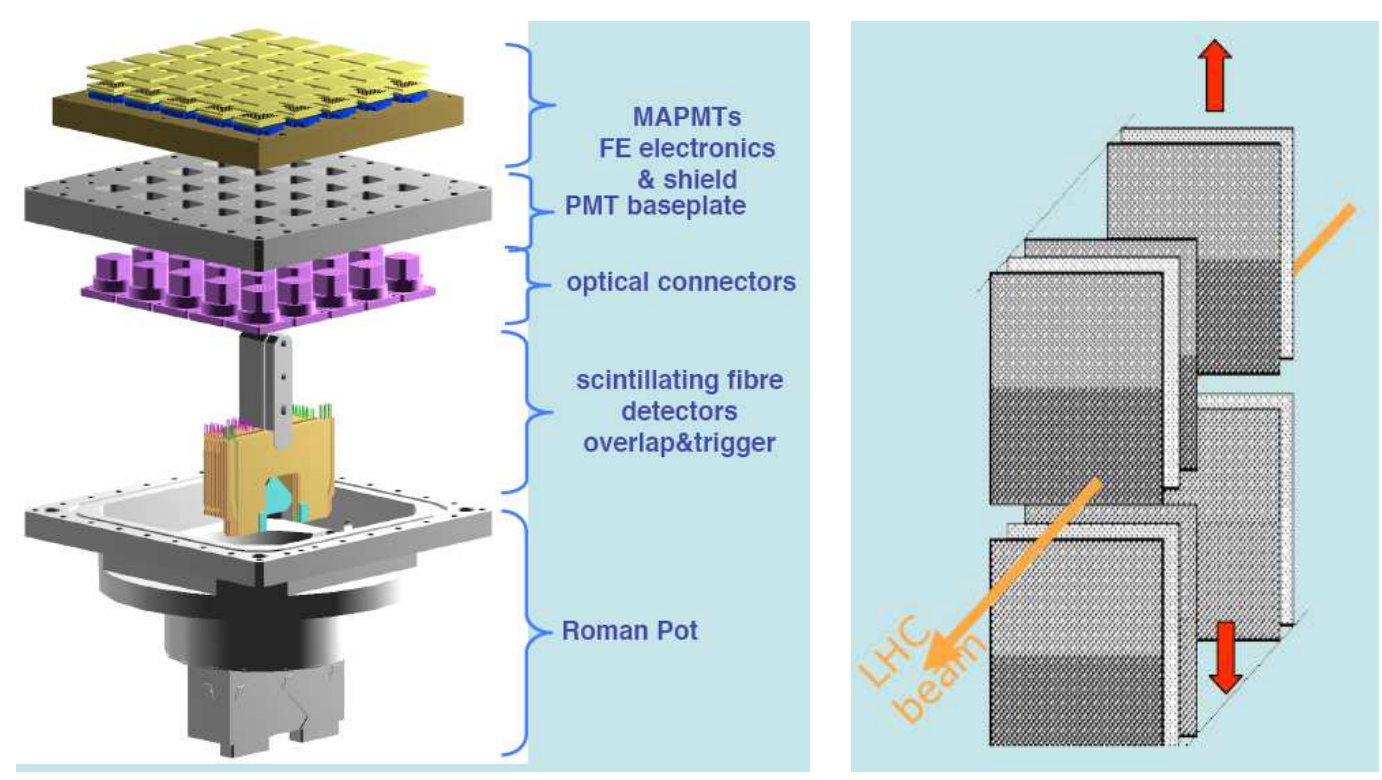

Figure 1: Scheme of the detector to be installed in the roman pots for luminosity meaurements

\subsection{Roman pot detectors}

The project is to install two sets of roman pot detectors on each side of ATLAS located at about $240 \mathrm{~m}$ from the interaction point. Each pot is itself made of two detectors in the vertical direction. The roman pot design is inspired by the TOTEM collaboration [3] and the changes with respect to TOTEM is that there is no horizontal roman pots (the acceptance for elastic events is small in the horizontal direction), and the geometry of flanges where the roman pots are mounted as well as the bases were modified to allow a different beam height with respect to TOTEM.

The detector to be installed inside the roman pots is described in Fig. 1. The detector made of $20 \times 64$ square $0.5 \times 0.5 \mathrm{~mm}^{2}$ scintillating fibers on ceramic substrate is read out by 24 Multianode photomultipliers with 64 channels. The detector follows a U/V geometry with 45 degree stereo layers, 64 fibers per plane in a module, 10 double sided modules per pot. The up and down detectors overlap for relative alignment purposes.

A prototype of the detector made of $20 \mathrm{U}$ and $\mathrm{V}$ planes of 6 fibers has been built and put into a test beam facility at DESY using $6 \mathrm{GeV}$ electron beams. The aims of the beam test were to study the photoelectric yield, the efficiency of the detector (especially at the edge), the cross talk between channels and the track resolution. The multiphoton spectrum is shown in Fig. 2. The fit to this spectrum is a two step process: first the position and width of the pedestal is fitted by a Gaussian and second, the contribution from 0 to 12 photoelectrons is fitted using a convolution between a Poisson distribution and a Gaussian, The average number of photoelectrons has been found to be 4.1 and the cross-talk between 3 and $4 \%$. The single fiber efficiency was found to be between 90 and $94 \%$, and the space resolution at LHC beam energies is expected to be of the order of $20 \mu \mathrm{m}$. The insensitive area at the edge of the detector is less than $30 \mu \mathrm{m}$. Of course some tests using higher energy beams will be necessary. 


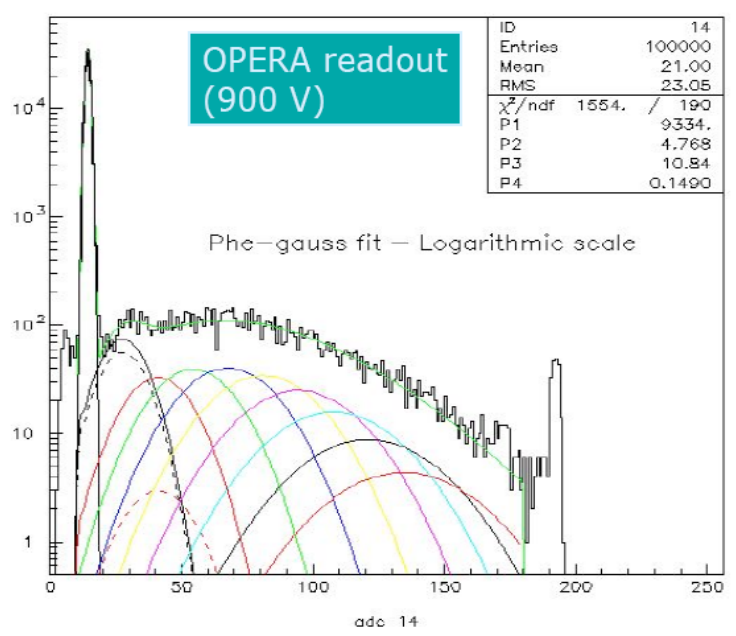

Figure 2: Multiphoton spectrum using the scintillating fiber detector measured during the electron beam tests at DESY

\begin{tabular}{|c||c||c|c|c|}
\hline Parameters & input & fitted & error & correlation \\
\hline \hline$L$ & $8.12410^{26}$ & $8.16210^{26}$ & $1.5 \%$ & \\
$\sigma_{\text {tot }}$ & $100 \mathrm{mb}$ & $101.1 \mathrm{mb}$ & $0.74 \%$ & $99 \%$ \\
$b$ & $18 \mathrm{GeV}^{-2}$ & $17.95 \mathrm{GeV}^{-2}$ & $0.59 \%$ & $64 \%$ \\
$\rho$ & 0.15 & 0.1502 & $4.24 \%$ & $92 \%$ \\
\hline
\end{tabular}

Table 1: Comparison between fitted parameters and input ones showing the accuracy of the luminosity measurement using the Coulomb method.

\subsection{Simulation of elastic scattering}

A full simulation of elastic events in a real detector has been performed to check the accuracy of the luminosity measurement within ATLAS. Two different values of $t$ have been used: $t=7 \cdot 10^{-4}$ and $t=10^{-3} \mathrm{GeV}^{2}$. The measured $t$ distribution for the 10 million simulated events is shown in Fig. 3, and a fit of this distribution using the following formula has been performed:

$$
\frac{d N}{d t}=L\left(\frac{4 \pi \alpha^{2}}{|t|^{2}}-\frac{\alpha \rho \sigma_{t o t} e^{-b|t| / 2}}{|t|}+\frac{\sigma_{t o t}^{2}\left(1+\rho^{2}\right) e^{-b|t|}}{16 \pi}\right) .
$$

The comparison between fitted parameters and input ones is given in Table 1 . We note that a small difference is obtained between both numbers with the caveat that the statistical correlations between the luminosity and the other parameters are large.

\subsection{Relative luminosity measurement using LUCID}

The relative luminosity measurement as a function of time will be done in ATLAS using the LUCID detector [1] (Luminosity measurement Using Cerenkov Integrating Detectors). The front face of the LUCID detector is located at about 17 meters from the ATLAS interaction point and 


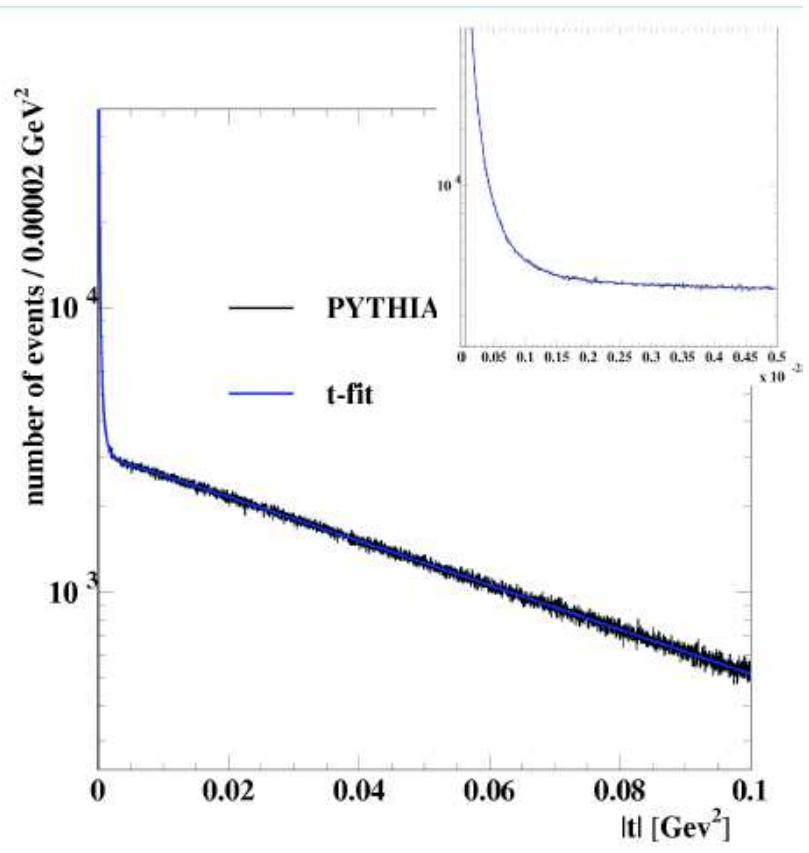

Figure 3: $d N / d t$ distribution corresponding to the simulation of 10 million elastic events using PYTHIA.

covers a domain in rapidity of $5.4<|\eta|<6.1$. The principle of the LUCID detector is shown in Fig. 4. 168 Aluminium tubes are filled with $C_{4} F_{10}$ or isobutane at 1 or 2 bar pressure. Winston cones at the end of each tube bring the Cerenkov light onto quarz fibers. It is thus possible to measure the number of particles which are produced in the very forward region which is directly related to the instantaneous luminosity. The LUCID detector is mainly sensitive to primary particles only: much more light is coming from primary particles than from secondaries or soft particles. The time resolution of this detector is of the order of $140 \mathrm{ps}$ (following CDF measurements [4]) which allows to determinate the luminosity bunch by bunch at the LHC. This detector allows to obtain a linear relationship between the luminosity and the number of tracks counted in the detector which leads to an easy measurement of the luminosity. The amplitude resolution is excellent, it is possible to count even multiple tracks per tube and no saturation is observed even at highest luminosity. Beam tests lead to an average number of photoelectrons per Cerenkov tube of the order of 5.3, which is a bit lower than expected. Further improvements such as the coupling between the fiber and the tube are in progress.

\section{Hard diffraction in ATLAS}

We will now discuss the project of building detectors sensitive to hard diffraction in ATLAS. Whereas the luminosity measurements and the LUCID detectors are well established, the option to install detectors to detect diffractive events at the highest luminosity is just starting and being discussed within ATLAS. 


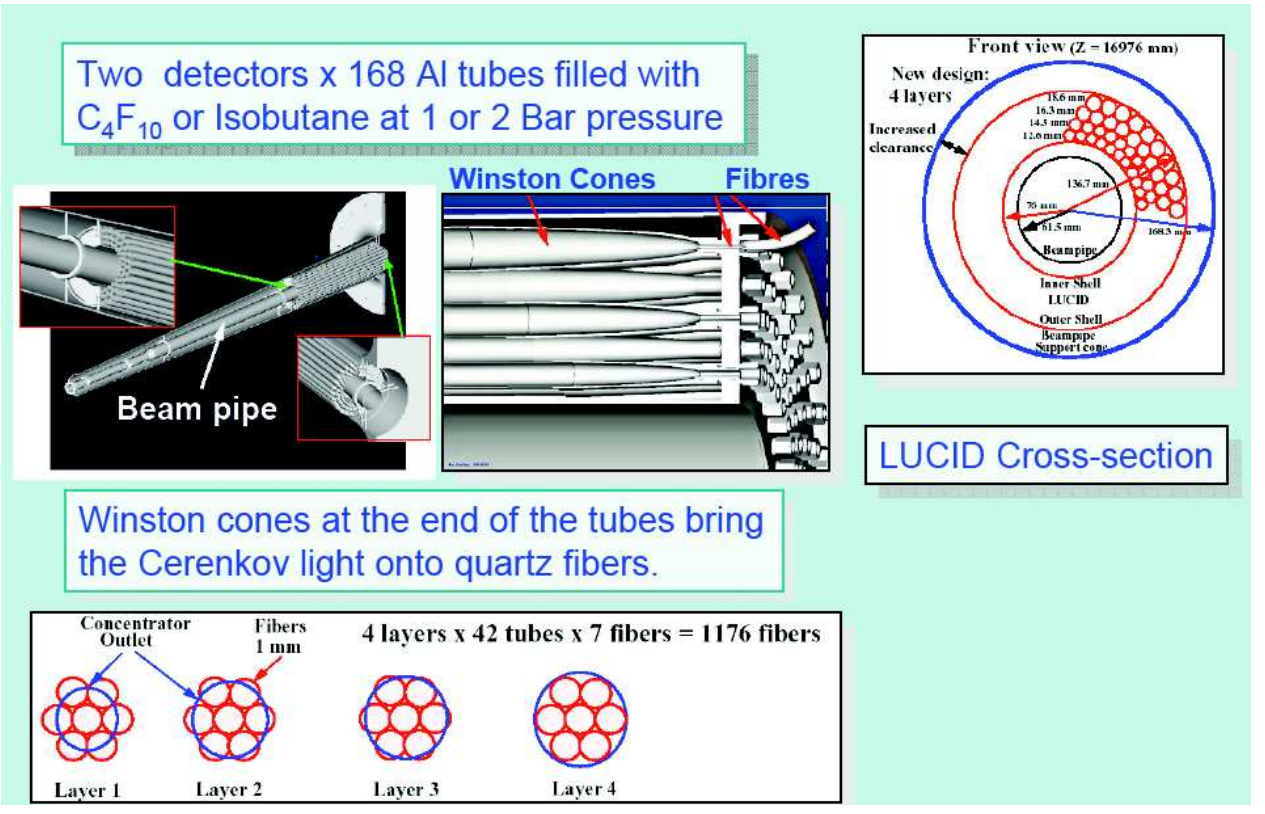

Figure 4: Scheme of the LUCID detector.

The main motivation to install roman pot detectors working at the highest luminosity is the search for the Higgs boson [5], supersymmetric events [6] and usual QCD studies such as the measurement of the gluon density in the pomeron. Two options are considered within ATLAS: installing roman pot detectors at $420 \mathrm{~m}$ (the FP420 project [7]) and at $220 \mathrm{~m} \mathrm{[8].} \mathrm{In} \mathrm{this} \mathrm{talk,} \mathrm{we}$ will only mention the second one. Roman pot detectors will be installed at 216 and 224 meters from the ATLAS interaction points. The detectors are placed in a TOTEM-like roman pot with an horizontal arm only. They need to show little dead edges to be able to be sensitive to the lowest Higgs mass possible, and a good space resolution is needed to get a good resolution on mass. A very good timing of the order of $5 \mathrm{ps}$ is also needed to determine from which vertex the diffracted protons are coming since up to 25 interactions per bunch crossing can occur at the LHC at the highest luminosity.

After a full simulation of the beam to compute beam spots and the implementation of the beam optics, the acceptance of the roman pot detectors at $220 \mathrm{~m}$ can be computed. The results are shown in Fig. 5. The left plot of Fig. 5 shows the position of the diffracted protons with $|t|=0$ (center of circles) or $|t|=0.05 \mathrm{GeV}^{2}$. The different circles from left to right show the $\xi$ dependence by steps of 0.02 for the two stations at 220 and $240 \mathrm{~m}$ where $\xi$ is the fraction of the proton momentum carried by the pomeron. The right plot of Fig. 5 shows the same distribution zooming in the low $\xi$ region. The two lines show the lowest values in $\xi$ obtained if the detector can be put at 10 or $20 \sigma$ from the beam. This shows that it is possible to reach a Higgs mass down to $125 \mathrm{GeV}$ using roman pots at $220 \mathrm{~m}$ only provided it is possible to put the detectors at $10 \sigma$ from the beam.

The detectors will be made of two different parts. Nine layers of Silicon stip detectors of 50 $\mu \mathrm{m}$ will provide the needed space resolution of the order of 10 to $15 \mu \mathrm{m}$. Two planes will be 

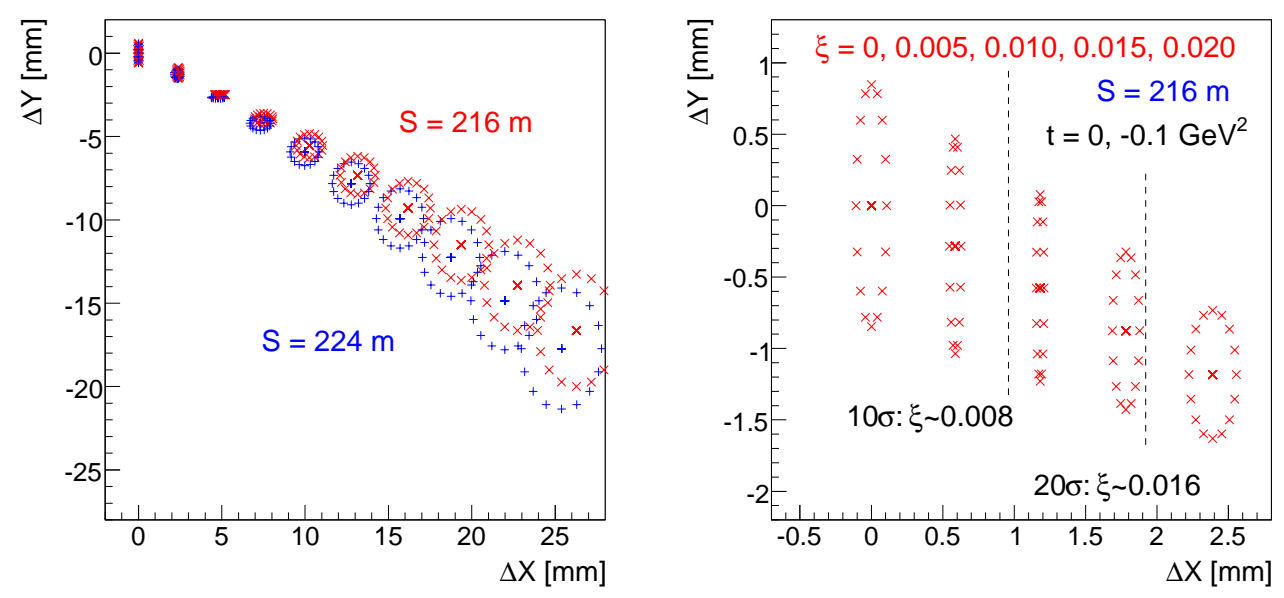

Figure 5: Acceptance for roman pot detectors to be installed at $220 \mathrm{~m}$ (see text).

read out to be used directly at the first level of the ATLAS trigger which will look for coincidences between two planes from the same roman pots and between the two roman pots as well. The dead material at the edge will be less than $30 \mu \mathrm{m}$ to minimize the dead zone at the edge of the detector. The readout of the detectors should be of the order of 5 ns to know from which bunch crossing the protons are coming. A collaboration between Prague, Stony Brook, Cracow and Saclay is being formed to buid the roman pots and their detectors.

In addition to the Silicon strip detectors, very precise Cerenkov timing detectors with a resolution better than $5 \mathrm{ps}$ will be installed. The needed precision on the vertex position using these detectors is of the order of $1 \mathrm{~mm}$ (note that the difference in distance between two protons coming from the same vertex but having a different trajectory in the magnet system, or in other words showing different $\xi$ or $t$ is of the order of $50 \mu \mathrm{m}$, much smaller than the precision of $1 \mathrm{~mm}$ we want to reach). Such timing detectors are being developped for this purpose and also for medical imaging applications. A collaboration between the University of Chicago, Argonne National Laboratory and CEA Saclay is being developped following these lines.

\section{Conclusion}

In this article, we have described the measurement of the elastic scattering in the Coulomb region which will be used by ATLAS to measure the absolute luminosity, as well as the LUCID detectors which allow to make a measurement of the relative luminosity. We also describe the project to build new roman pots at 220 meters and their Silicon strip and precise timing detectors allowing a resolution of $15 \mu \mathrm{m}$ and 5 ps respectively.

\section{References}

[1] ATLAS forward detectors for luminosity measurement and monitoring, Letter of Intent, CERN/LHCC/2004-010. 
[2] UA4 Coll., Phys. Lett. B198 (1987) 583.

[3] Totem TDR, CERN/LHCC/2004-002.

[4] CDF Coll., Nucl. Inst. and Meth. A461 (2001) 540; Nucl. Inst. and Meth. A494 (2002) 57.

[5] C. Royon, Mod. Phys. Lett. A 18, 2169 (2003) and references therein; M. Boonekamp, R. Peschanski, C. Royon, Phys. Rev. Lett. 87 (2001) 251806 and Nucl. Phys. B669 (2003) 277; M. Boonekamp, A. De Roeck, R. Peschanski, C. Royon, Phys. Lett. B550 (2002) 93; V.A. Khoze, A.D. Martin, M.G. Ryskin, Eur. Phys. J. C19 (2001) 477 and Eur. Phys. J. C24 (2002) 581.

[6] M. Boonekamp, J. Cammin, S. Lavignac, R. Peschanski, C. Royon, Phys. Rev. D73 (2006) 115011 and references therein; M. Boonekamp, J. Cammin, R. Peschanski, C. Royon, preprint hep-ph/0504199.

[7] FP420 Coll., see http://www.fp420.com.

[8] Letter of Intent for the $220 \mathrm{~m}$ roman pot project in ATLAS. 